\title{
The Flame-Retardant Performances of Blending Fabrics of Flame-Retardant Viscose and Nylon 6, 6 Fiber with Different Blending Ratio
}

\author{
Dr. Noopur Sonee*, Dr. Chita Arora*, Dr. M.S. Parmar** \\ *(Department of Fabric and Apparel Science, Delhi University, Delhi, India \\ ** (Northern India Textile Research Association, Ghaziabad, India
}

\begin{abstract}
In this article the fire-retardant viscose and the nylon 6, 6 fibers were used to produce blends and wove the flame-resistant fabrics with different blending ratios, then tested and analyzed the flame-resistant performance with the Limiting Oxygen Index, Radiation Heat Transfer Index and the Limited Flame Spread (Surface and Edge ignition) test methods. The experimental results are that the blending fabrics of the fire-retardant viscose and the nylon 6, 6 have good fire resistance. Comfort properties were also determined good of blended fabrics. It is of an important practical significance to develop flame-retardant fabric.
\end{abstract}

Keywords: Flame-Retardant Viscose, Nylon 6, 6, Blends, Comfort, LOI

\section{INTRODUCTION}

Flame-Retardant viscose: It is produced by incorporating FR additives/fillers in the spinning dope before extrusion. During burning of this fiber the flame point produce a lot of nitrous oxide, which effectively isolates the fiber flame point from oxygen, thereby showing a fire-retardant effect [1]. This fiber does not show combustion and will instantly extinct without fire source with low smoke concentration. This fiber when blended with polyester fiber provides special protection. It shows small thermal shrinkage which avoids second burn injury.

Nylon 6, 6: It is an aliphatic thermoplastic prepared by polycondensation of adipic acid and hexamethylenediamine [2]. The melting point of this polymer is $268^{\circ} \mathrm{C}$, which is high for synthetic fibers. This property makes it heat and friction resistance and also exhibits high abrasion resistance and selflubricating properties. It has high thermal stability, fire resistance, drawability, good appearance and good process ability.

The fire-retardant viscose and nylon 6, 6 fibers were used to produce blends with $20^{\mathrm{s}}$ count yarn and 16 TPI with different ratio such as 30:70, 50:50 and 70:30 using ring spinning system and converted into plain rip-stop woven fabrics and their fire-resistance and comfort properties were studied. Percent of fiber blends and the sample codes can be seen in Table 2. Under the past experiences and theoretical basis for fiber, blended yarn and physical properties, comfort properties of fabrics and fireretardant properties were systematically studied, analyzed and discussed.

\section{EXPERIMENTAL MATERIAL}

Material: The properties of fire-retardant viscose and nylon 6, 6 fibers are shown in Table 1 .

Table 1: Physical properties of fiber

\begin{tabular}{lll}
\hline $\begin{array}{l}\text { Fiber } \\
\text { Properties }\end{array}$ & Nylon 6,6 & FR Viscose \\
\hline Length (mm) & 38 & 51 \\
Density (denier) & 1.60 & 1.98 \\
Tenacity (g/tex) & 48.51 & 24.21 \\
Elongation (\%) & 27.46 & 13.78 \\
\hline
\end{tabular}

To produce yarns blending was done during blow room stage. Spindle speed of the ring frame was kept at $8000 \mathrm{rpm}$. Prepared yarn samples were tested for their physical properties using standard test procedures under atmospheric conditions, which are discussed in Table 3. Developed yarns were converted into plain rip stop woven fabrics (Ends/inch: 80 and Picks/inch: 60) using rapier loom.

Table 2: Blend ratio of fibers in yarn samples

\begin{tabular}{lcc}
\hline Fiber & $\begin{array}{c}\text { Sample } \\
\text { Code }\end{array}$ & $\begin{array}{c}\% \text { Fiber } \\
\text { Blend } \\
\text { Ratio }\end{array}$ \\
\hline FR Viscose & V1 & 100 \\
Nylon 6, 6 & N1 & 100 \\
FR Viscose: Nylon 6, 6 & VN37 & $30: 70$ \\
FR Viscose: Nylon 6, 6 & VN55 & $50: 50$ \\
FR Viscose: Nylon 6, 6 & VN73 & $70: 30$ \\
\hline
\end{tabular}



ISSN : 2248-9622, Vol. 7, Issue 6, ( Part -6) June 2017, pp.87-91

Table 3: Physical properties of developed yarns

\begin{tabular}{lccc}
$\begin{array}{c}\text { Sample } \\
\text { Code }\end{array}$ & $\begin{array}{c}\text { Tenacity } \\
(\mathrm{g} / \mathrm{tex})\end{array}$ & $\begin{array}{c}\text { Elongation } \\
(\%)\end{array}$ & $\begin{array}{c}\text { Count } \\
\text { Strength } \\
\text { Product } \\
\text { (CSP) }\end{array}$ \\
\hline V1 & 13.1 & 7.8 & 2121 \\
N1 & 26.1 & 21.0 & 3843 \\
VN37 & 22.29 & 21.43 & 3024.1 \\
VN55 & 19.15 & 11.65 & 2905.9 \\
VN73 & 16.95 & 8.40 & 2489.4 \\
Key: V- FR viscose, N- Nylon 6,6
\end{tabular}

ISO 6330, 2A washing method was followed for washing the fabrics in automatic washing machine (front loaded) at $60^{\circ} \mathrm{C} \pm 3$ for 30 minute for completing one cycle along with $2 \pm 0,1 \mathrm{~kg}$ total load. Agitation was kept normal during heating, washing and rinsing. AATCC Standard Reference Detergent was used for washing [3]. After washing fabrics were tumble dried. Finally, the fabric samples were tested and evaluated using standard test methods for various physical properties, comfort and heat and flame properties which are given below:

- Air Permeability (IS 11056: 1984)

- Water Vapour Permeability (ISO: 15496)

- Limiting Oxygen Index (IS: 13501)

- Radiation Heat Transfer Index (ISO: 6942)

- $\quad$ Limited Flame Spread (ISO: 15025)

The results of these fabrics were also compared with the specified values given in the specification ISO 11612: Protective clothingClothing to protect against heat and flame.

\section{RESULTS AND DISCUSSION}

Physical properties of developed yarn:

N1 demonstrated higher tenacity i.e. 26.10 g/tex and CSP i.e. 3843 which was comparatively more than other yarn samples. Since V1 demonstrated lower tenacity. Hence, Viscose/ nylon 6,6 blended sample in ratio of 30:70 (VN37) showed lower tenacity as compared to N1 (100\% nylon 6,6). Fabric VN55 and VM73 found with comparatively lower tenacity on decreasing the percent of Nylon. 6, 6 . Elongation of N1 was $21 \%$, while V1 showed lower percent of elongation as compared to N1 (Table 2).VN37 showed approximately same value as $100 \%$ Nylon 6, 6, while VN55 and VN73 presented lower elongation.

Physical properties of developed fabrics:

It is clear from the Table 4 that GSM of V1, $\mathrm{N} 1, \mathrm{VN} 37, \mathrm{VN} 55$ and VN73 are $194 \mathrm{~g} / \mathrm{m}^{2}, 204 \mathrm{~g} / \mathrm{m}^{2}$, $189 \mathrm{~g} / \mathrm{m}^{2}, 194 \mathrm{~g} / \mathrm{m}^{2}$ and $187 \mathrm{~g} / \mathrm{m}^{2}$ respectively. $\mathrm{N} 1$ showed highest fabric weight as compared to other fabrics. V1, N1, VN37, VN55 and VN73 fabrics were determined with thickness of $0.50 \mathrm{~mm}, 0.75 \mathrm{~mm}, 0.48$ $\mathrm{mm}, 0.51 \mathrm{~mm}$ and $0.52 \mathrm{~mm}$ respectively. It is clear from the Table 4 that $\mathrm{N} 1$ was found thicker $(0.75$ $\mathrm{mm})$ as compared to other fabrics.

Air permeability: It was determined using WIRA Air Permeability Tester following IS 11056: 1984 test method. This test indicated the amount of air passing through test specimen on the scale reading, in terms of $\mathrm{cc} / \mathrm{cm}^{2} / \mathrm{sec}$. Results presented in Fig. 1 shows that V1 $\left(28.1 \mathrm{cc} / \mathrm{sec} / \mathrm{cm}^{2}\right)$ presented highest air permeability, while N1 (100\%) presented lowest air permeability due to the fact of highest thickness.

Air permeability is mainly dependent upon the fabric's weight and construction (thickness and porosity) as well as many factors such as fiber fineness, structural properties such as shape and value of pores of the fabric and the yarn [4] [5]. FR Viscose/nylon 6, 6 blended fabrics VN37, VN55 and VN73 were determined with reduced air permeability on reducing the percent of FR viscose (Table 4).

Water vapour permeability: The resistance to the flow of air and heat both affect comfort provided by a fabric used as clothing. A third such quantity is the resistance to the diffusion of water vapour across the fabric. For comfort the permeability to water vapour of clothing fabric should be as high as possible to allow the escape of water vapour which

Table 4: Properties of developed Fabrics

\begin{tabular}{|c|c|c|c|c|c|c|c|c|}
\hline \multirow[t]{2}{*}{ Prox } & \multirow[t]{2}{*}{$\begin{array}{l}\text { lidaxs } \\
\text { (II) }\end{array}$} & \multirow[t]{2}{*}{$\begin{array}{l}\text { Wegit } \\
(5 \mathrm{IF})\end{array}$} & \multirow{2}{*}{ 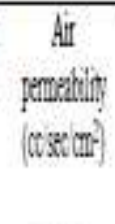 } & \multirow{2}{*}{ 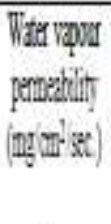 } & \multicolumn{2}{|c|}{ 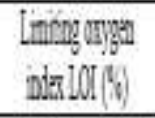 } & \multicolumn{2}{|c|}{$\begin{array}{c}\text { Refing ies inder } \\
\text { (xi) }\end{array}$} \\
\hline & & & & & Atri & & $\begin{array}{l}\text { Afte: } \\
\text { roishs }\end{array}$ & \\
\hline & 18 & 144 & $\overline{81}$ & 100 & 165 & 271 & 121 & 21 \\
\hline & 2 & X & 10 & 8 & 1118 & 118 & & 12 \\
\hline & H & 18 & 17. & 11 & 130 & 115 & 123 & 12 \\
\hline & U) & 194 & $19 !$ & 11. & b.) & 16.0 & 131 & \\
\hline & 03: & 187 & 213 & 120 & 260 & 15. & 124 & 12 \\
\hline
\end{tabular}

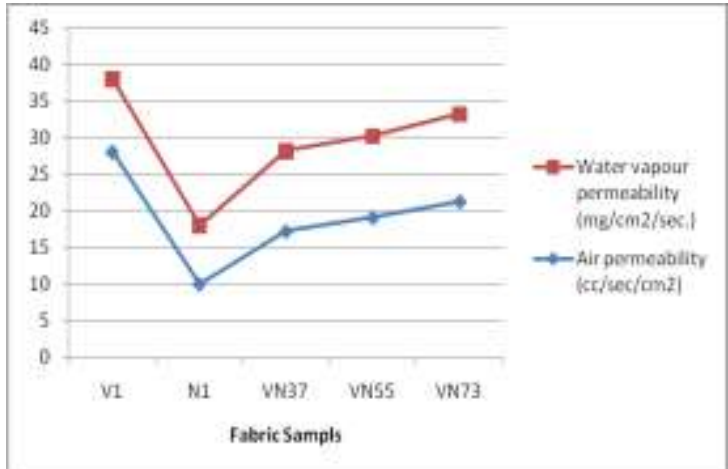

Fig. 1. Air permeability and Water vapour permeability of developed Fabrics 
is being released from the skin, even when the person is inactive [6].

It was measured on Water Vapour Permeability Tester: (M261) by SDL International Ltd following ISO: 15496 test method. Results presented in Fig. 1 shows that V1 and N1 displayed water vapour permeability of $10 \mathrm{mg} / \mathrm{cm}^{2} / \mathrm{second}$ and $8 \mathrm{mg} / \mathrm{cm}^{2} / \mathrm{sec}$, respectively. On the contrary, FR viscose/nylon 6, 6 blended fabrics VN37, VN55 and VN73 presented better water vapour permeability of $11 \mathrm{mg} / \mathrm{cm}^{2} / \mathrm{sec}, 11.2 \mathrm{mg} / \mathrm{cm}^{2} / \mathrm{sec}$ and $12 \mathrm{mg} / \mathrm{cm}^{2} / \mathrm{sec}$, respectively.

According to CRPF specification on NYCO fabric, minimum requirement of air permeability is three (3) $\mathrm{cc} / \mathrm{sec} . \mathrm{cm}^{2}$ and minimum requirement of water vapour permeability is given five (5) $\mathrm{mg} / \mathrm{sec} . \mathrm{cm}^{2}$. According to DEBEL specifications, FR overalls should pass $20 \mathrm{cc} / \mathrm{sec} . \mathrm{cm}^{2}$ air permeability as a minimum requirement for comfort [7][8].

It is clear from the above Table 4, all the fabrics showed better air permeability and water vapour permeability as compared to the requirement given in CRPF and DEBEL specifications.

Limiting Oxygen Index (LOI): It was measured by passing a mixture of oxygen and nitrogen gases over a burning specimen and reducing the oxygen level until a critical level is reached [9]. Fabric with LOI < 21.0 are considered very flammable, those with LOI 21.0-25.0 are moderately flammable and generally if LOI > 25.0, textiles start to pass various standard tests for flame retardant textiles [10].

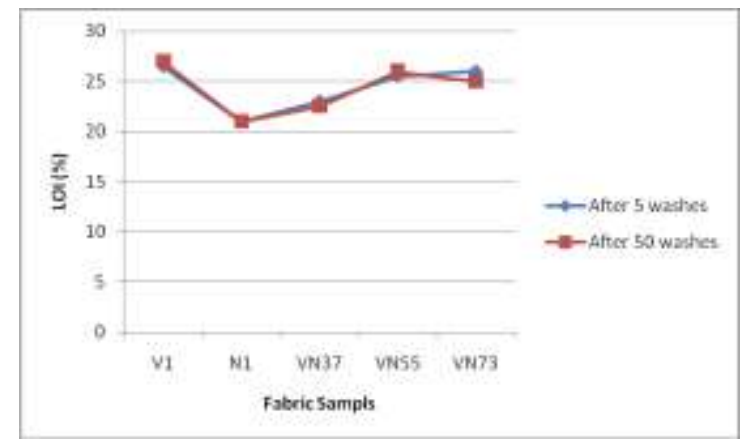

Fig. 2. Limiting oxygen index

V1 had $26.5 \%$ LOI, while N1 had much lower LOI (21\%), therefore it burns rapidly. LOI results clearly showed that on increasing the percent of FR viscose with nylon 6, 6, LOI \% also increased in the blended fabrics such as VN37 had 23\%, VN55 had $25.5 \%$ and VN73 had $26 \%$ LOI. After 50 washes all fabrics showed minimal change in LOI (Fig. 2).

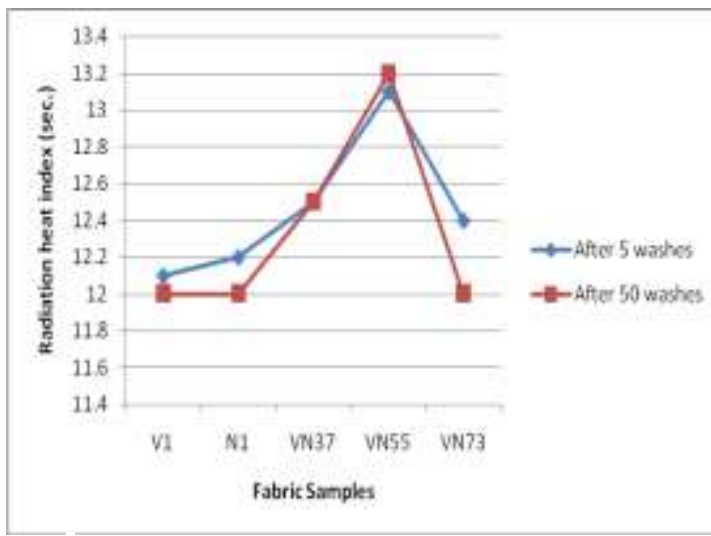

Fig. 3. Radiation heat transfer index

Radiation heat transfer index: Fabrics were exposed to radiation heated source emitting 20 $\mathrm{kW} / \mathrm{m}^{2}$ heat fluxes following ISO 6942 test method. According to Fig. 3, approximately similar results were seen in all fabrics excluding VN55, presented the highest threshold time (13.1 seconds). Threshold time for radiation heat source for $\mathrm{N} 1$ fabric was found similar as V1; the reason could be the higher thickness and weight of $\mathrm{N} 1$ fabric. The radiant protective performance of single-layer fabric is affected by the structure, weight, thickness and materials [11][12]. After 50 washes all fabrics showed very less changes. According to ISO11612, all fabric samples showed better radiation heat index than the minimum requirement of 7 seconds.

Limited flame spread: Fabrics were evaluated for surface and edge ignition. To meet the criteria of surface ignition (Table 5), fabric samples were observed whether any flaming reaches the top edge or either side edge of fabric, whether a hole develops, occurrence of flaming melting debris, after flame time and afterglow time. For edge ignition (Table 6), fabric samples were observed whether any flaming reaches the top edge or either side edge, occurrence of flaming melting debris, after flame time and afterglow time following ISO 11612 International standard for protective clothing.

Table 5 and 6 explains that all samples V1, VN55, VN73 passed the criteria for surface ignition as well as edge ignition as mentioned in the ISO 11612 specification even after 50 washes. While nylon 6, $6100 \%$ and FR viscose/ nylon 6, 6 blend $(30 / 70)$ could not even meet the minimum requirements given in ISO 11612 standard even after 5 washes. $100 \%$ nylon 6, 6 showed less after flame and after glow time as compared to FR viscose/nylon 6 , 6 blend (VN37), the reason could be the higher thickness and weight of N1 (Table 4). The physical properties of the fabric include its mass, construction and configuration. Mass per unit area is directly proportional to the ease of ignition and linear burning rate. Denser and heavier the fabric, longer it takes to ignite and longer it takes to bum [1]. 


\section{CONCLUSION}

Through the analysis of the experimental process and experimental results, it was concluded that nylon 6, 6 has good strength and Flame-retardant viscose has good permeability and flame -retardancy. The flame-retardancy of the combination fabrics with fire-retardant viscose and nylon 6, 6 is strengthened with the percentage of nylon 6, 6 increases.

$100 \%$ Nylon 6, 6 does not have good permeability, while blended fabrics of FR viscose and nylon 6, 6 shows good comfort properties. The LOI of fabrics rose with FR viscose percentage, the higher content of FR viscose the better flame-retardancy of fabrics. Considering fabric performance, when the fire-retardant viscose and nylon 6, 6 blending ratio is 50:50 and 70:30, the fabric not only have good fire resistance performance but also can satisfy the human requirements in aspects of comfort and serviceability.

From the study it is clear that FR viscose and nylon 6, 6 blends in ratio of 50:50 and 70:30 respectively were found better as well as meeting all the requirements of ISO 11612. This study will guide the manufacturers to select suitable fiber blends to make the product cost effective without compromise the minimum requirement as mentioned in the ISO 11612.

\section{ACKNOWLEDGEMENTS}

This work was financially supported by University Grant Commission in form of research fellowship - JRF. We are also grateful to Northern India Textile Research Association for their support.

Table 5: Limited flame spread (Surface Ignition)

\begin{tabular}{|c|c|c|c|c|c|c|}
\hline \multicolumn{2}{|l|}{ Surface Ignifin } & \multirow{2}{*}{ 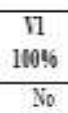 } & \multirow{2}{*}{$\begin{array}{r}\begin{array}{c}\mathrm{N} 1 \\
106 \%\end{array} \\
\mathrm{Yes}\end{array}$} & \multirow{2}{*}{ 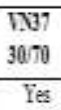 } & \multirow{2}{*}{$\begin{array}{r}1 \mathrm{X55} \\
5150 \\
\mathrm{No}\end{array}$} & \multirow{2}{*}{$\begin{array}{r}\text { IN73 } \\
7436 \\
\mathrm{No}\end{array}$} \\
\hline \multirow{2}{*}{$\begin{array}{l}\text { Whether any flaming } \\
\text { Irashes the top etge or } \\
\text { ther side edge }\end{array}$} & After 5 mashes & & & & & \\
\hline & Aftar 52 wahies & No & Yes & Yes & $\mathrm{No}_{0}$ & No \\
\hline \multirow{2}{*}{$\begin{array}{l}\text { Whether a hole } \\
\text { derelpps }\end{array}$} & Atter 5 rashes & No & Yes & Yes & No & No \\
\hline & After Y0 wakhes & No & Yes & Yes & No & $\mathrm{No}$ \\
\hline \multirow{2}{*}{$\begin{array}{l}\text { Ocumence of flaming } \\
\text { mating debris }\end{array}$} & After 5 mashes & No & $\mathrm{Y}_{e s}$ & No & $\mathrm{Ne}$ & No \\
\hline & After $\%$ wathes & No & Yes & $\mathrm{No}_{0}$ & $\mathrm{Ne}$ & No \\
\hline \multirow{2}{*}{ After flametime (sec) } & After 5 mashes & $\mathrm{M}$ & 25 & 818 & $\mathrm{Mi}$ & 国 \\
\hline & Aftar 5\% washes & $\mathrm{NI}$ & 21 & 52 & $\mathrm{M}$ & $\mathrm{s}$ \\
\hline \multirow{2}{*}{ After glvw fime (sec) } & Atter 5 mashes & $\mathrm{Ni}$ & Ni & $\mathrm{N}$ & Yi & Mi \\
\hline & After 50 vathes & $\mathrm{MI}$ & Ni & N & Si & $\mathrm{Gi}$ \\
\hline \multicolumn{7}{|c|}{ As per 1S0 11612:Limitad flame spresd (Strface iggition) } \\
\hline \multicolumn{7}{|c|}{ 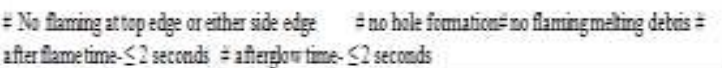 } \\
\hline
\end{tabular}

Table 6: Limited flame spread (Edge Ignition)

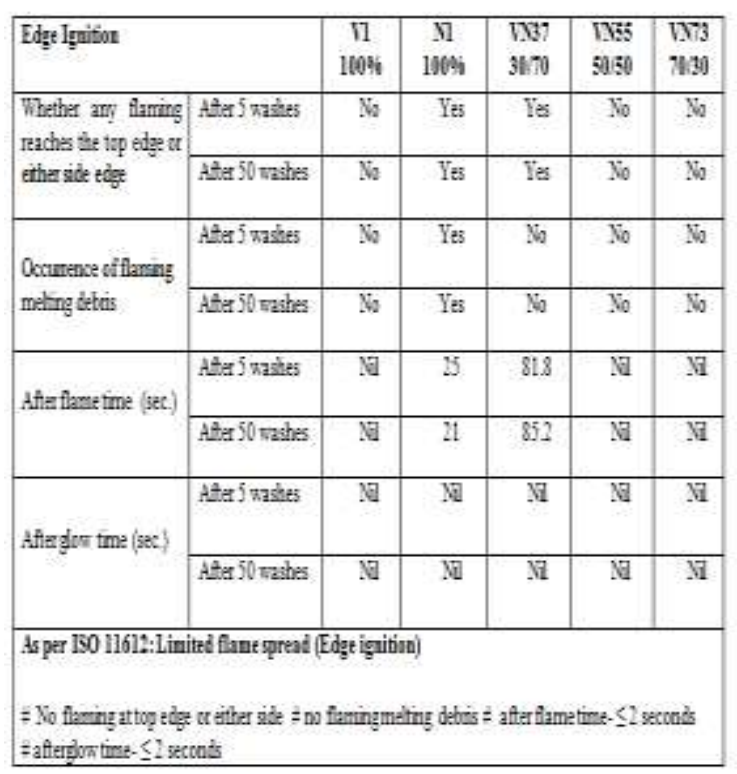

\section{REFERENCES}

[1] A.R. Horrocks, and S.C. Anand (Eds.) Handbook of Technical Textile (Washington, DC Woodhead Publishing Limited, 2000).

[2] Michael J. Troughton (Eds.) Handbook of Plastics Joining: A Practical Guide (Norwich, NY William Andrew Inc, 2008).

[3] Tugrul, R. Ogulata. Air permeability of woven fabrics. Journal of Textile and Apparel Technology and Management, 5 (2), 2006, 1-2.

[4] ISO 6330, Textiles- Domestic washing and drying procedure for textile testing

[5] J. Y. Hu, Y. Li, and K. W. Yeung. Air Permeability. Y. Li. and A.S.W. Wong (Eds.), Clothing Biosensory Engineering, pp: 252-260, (Cambridge, Woodhead Publishing Limited, 2006).

[6] V. K. Kothari. Thermo-physiological comfort characteristics and blended yarn woven fabrics. Indian Journal of Fiber \& Textile Research, 31, 2006, 177-186.

[7] CRPF specification on NYCO fabric, Retrieved from http://mha1.nic.in/QRs/clothings/QRSNylon-Cobra-19Oct2015.PDF, (2016, May).

[8] DEBEL Specifications Appendix -B (DEBEL/47/06 issue -B)

[9] IS: 13501: 1992, Indian Standard, Textilesdetermination of flammability by oxygen index

[10] A.R Horrocks, M. Tune and D. Price. The burning behavior of textiles and its 
assessment by oxygen-index methods.

Textile Progress, 18, 1989, 1.

[11] G. Sun, H.S. YOO, X.S. Zhang and N. Pan. Radiant protective and transport properties of fabrics used by wildland firefighters. Textile Research Journal, 70 (7), 2000, 567573.

[12] S. Zeinab, Abdel-Rehim, M.M. Saad, M. EIShakankery and I. Hanafy. Textile fabrics as thermal insulators. AUTEX Research Journal, 6 (3), 2006, 148-161 